\section{Case Reports in Oncology}

Case Rep Oncol 2019;12:421-425

DOI: $10.1159 / 00050067$

Published online: June 5, 2019

(C) 2019 The Author(s)

Published by S. Karger AG, Base

www.karger.com/cro

This article is licensed under the Creative Commons Attribution-NonCommercial 4.0 International License (CC BY-NC) (http://www.karger.com/Services/OpenAccessLicense). Usage and distribution for commercial purposes requires written permission.

\title{
Negativation of PD-L1 Postoperatively in Initially Inoperable Stage III Non-Small Cell Lung Cancer Treated with Pembrolizumab: Two Case Reports
}

\author{
Fadi Nasr ${ }^{a-c}$ Ahmad Al Ghoche ${ }^{a}$ Roland Eid $^{b}$ Lewis Nasr ${ }^{b}$ \\ Saada Diab ${ }^{a}$ Souheil Hallit ${ }^{c}$ Moussa Riachi ${ }^{d}$ \\ aHematology-Oncology Department, Mount Lebanon Hospital, Hazmieh, Lebanon; \\ ${ }^{b}$ Hematology-Oncology Department, Hotel-Dieu de France University Hospital, \\ Saint Joseph University, Beirut, Lebanon; 'Faculty of Medicine and Medical Sciences, \\ Holy Spirit University of Kaslik (USEK), Jounieh, Lebanon; dPulmonary Department, \\ Hotel-Dieu de France University Hospital, Saint Joseph University, Beirut, Lebanon
}

\section{Keywords}

Non-small lung cancer · Pembrolizumab · PDL-1 PDL-1 conversion

\section{Abstract}

Stage III non-small cell lung cancer is a border line stage between localized and metastatic disease. PDL-1 is gaining an important role in the therapeutic arsenal of lung cancer, the most frequent cancer worldwide. We report for the first time a negativation of PDL-1 status in 2 cases of stage IIIA NSCLC with conversion to operable disease after using immunotherapy. The first patient was a 59-year old female diagnosed incidentally to have stage IIIA inoperable NSCLC that was treated with combination chemo-immunotherapy, and converted to operable disease with a negative PD-L1 in the postoperative setting. The second case is that of a 56year old male that also had an inoperable stage IIIA NSCLC treated with chemotherapy first line followed by pembrolizumab at progression, then operated after surgical conversion, with negative PD-L1 postoperatively. In front of these findings, further work should be done to elucidate if the reverse of the PDL-1 status and the conversion to operability were due to the use of immunotherapy or to an incidental finding. If confirmed, it may have a therapeutic impact. 


\section{Introduction}

Lung cancer is leading cancer mortality and researches are ongoing to improve patient care and treatment efficacy [1]. Lung cancer can be divided into small cell and non-small disease. While little improvement was made in small cell lung cancer (SCLC), major improvement was seen in the management of locally advanced and metastatic non-small cell lung cancer (NSCLC) [2]. Immunotherapy with check point inhibitors, especially programmed death ligand (anti PDL-1), is currently leading innovation in cancer treatment with a major role in the management of metastatic lung cancer [3] and recently in the neoadjuvant setting in operable NSCLC [4]. Standard of care in stage III inoperable NSCLC is currently chemotherapy or concomitant chemoradiotherapy followed by maintenance immunotherapy with Durvalumab [5].

Hereby we describe the first two initially inoperable non-small cell lung cancer cases that were converted to operable after the use of immunotherapy, along with the negativation of PDL1 post-operatively.

\section{Case Report}

\section{Case1}

We hereby report the case of a 59-year-old female patient, smoker with chronic bronchitis, diabetes and dyslipidemia. In January 2017, she was found to have a slight infiltration at the apex of the right lung, incidentally while she was admitted for cholecystectomy. The results of a CT scan (computed tomography scan) showed a $21 \mathrm{~mm}$ nodule with multiple spiculations spreading in part towards the pleura with a slightly localized thickening. Multiple ganglia were detected in the right paratracheal and the pre-carinal regions, with the largest being of $2 \mathrm{~cm}$. The patient was lost to follow-up until April 2017 when a chest CT scan showed an increase to $2.5 \mathrm{~cm}$ in the largest diameter of the tumor with the presence of enlarged lymph nodes in the right paratracheal area, the barety lodge and the supra-aortic area, rendering the tumor inoperable. A bronchoscopy was negative and CT-guided biopsy of the mass revealed a primitive pulmonary adenocarcinoma (transcription terminator factor1 (TTF1) and cytokeratin 7 (CK7) positive), epidermal growth factor receptor (EGFR) and anaplastic lymphoma kinase (ALK) were negative, and PDL-1 significantly positive $(60 \%)$ using the clone Dako 22C3-Autostainer. EBUS (endobronchial ultrasound biopsy) confirmed the presence of a $2 \mathrm{~cm}$ pathologic right paratracheal lymph node (zone 4R). A positron emission tomography/ computed tomography scan (PET CT scan) showed a $2.5 \times 2.9 \times 2.4 \mathrm{~cm}$ mass with SUV (standardized uptake values) max of 10.4, with a large cluster of fluorodeoxyglucose (FDG) avid right paratracheal lymph nodes extending to the carina measuring $4.2 \mathrm{~cm}$ in length, SUV max 7.58. Patient was therefore classified as an inoperable stage IIIA (T2N2M0) according to the eight American joint committee on cancer (AJCC) classification. Four cycles of chemoimmunotherapy with pemetrexed $500 \mathrm{mg}$, carboplatin $300 \mathrm{mg}$ and pembrolizumab $200 \mathrm{mg}$ were given every 21 days between June 2017 and August 2017, after which a radiologic evaluation by CT scan in September 2017 showed a regression of the lobulated and spiculated nodule of the apical segment of the right upper lobe, measuring $22 \times 12 \mathrm{~mm}$ with decrease in lymph node size. An injected brain MRI (magnetic resonance imaging) was also negative.

After these results, disease was considered stable according to WHO (world health organization) and RECIST 1.1; a decision was therefore taken to continue 4 more cycles of the same chemo-immunotherapy protocol from September to November 2017. After that, a PET CT scan and injected chest CT showed a regression of the mass to $1.4 \mathrm{~cm}$ with SUV $2(72 \%$ 
regression of the tumor compared to initial investigation and a regression of the right paratracheal lymph node to $1.8 \mathrm{~cm}$ with SUV max of 5.3). It was therefore decided to continue the treatment with Pembrolizumab monotherapy. EBUS done in January 2018 showed no evidence of disease in lymph nodes.

Pembrolizumab monotherapy was continued until March 2018 where a PET CT scan showed a 1.6-cm mass with SUV max of 1.65. The right paratracheal region showed a stable active adenopathy, measuring $1.5 \mathrm{~cm}$ in diameter with a SUV max of 5.15. So the decision was to do right upper lobectomy in April 2018. The pathology report showed an almost complete regression of the carcinomatous proliferation at the level of the main tumor with persistence of a predominantly lepidic carcinomatous focus in the secondary nodule, and 11 negative lymph nodes. The PDL-1 on post-operative tissue was negative. The patient is still in remission 4 months after surgery.

Case 2

The second case is a 56-year old Lebanese male, smoker, that was found in April 2016 to have multiple soft tissue nodules in the left lung on CT scan, the largest of which measuring $1.5 \mathrm{~cm}$. Bronchial biopsy showed a metastatic epithelium of poorly differentiated adenocarcinoma (TTF1 and p63 positive) with rare carcinomatous non-small cells in bronchial wash. PET CT scan in April 2016 showed left upper lobe active mass with small active left hilar lymph node and centimetric adenopathy in the left para-aortic region, the latest were noted as inflammatory or secondary by the radiologist. Injected total body CT scan later on in April showed a 2-cm spiculated nodule in postero-apical segment of left lobe with intimate contact with left pulmonary artery (staged as T4) along with infiltration around the left main bronchus, $3 \mathrm{~cm}$ above the carina with no other associated abnormalities. Patient was then classified as IIIA (T4N1M0) inoperable NSCLC according to the eighth AJCC classification. 21 days cycles with Gemcitabine 1,300 mg day 1 and 8 and carboplatin $300 \mathrm{mg}$ day 1 were started and given for 7 cycles. After that, a chest CT scan was done in October 2016 and showed persistence of the previously noted inoperable spiculated mass in the left upper lobe with newly appearing hilar adenopathies with posterior extension reaching lung fissure along with new appearing $7 \mathrm{~mm}$ opacity in the right lung at the postero-basal segment. In front of this progression, PDL1 test was done using IHC staining for Abcam clone on the initial lung tissue and showed $80 \%$ PD-L1 expression. So the decision was to start second line therapy in November 2016 with pembrolizumab $200 \mathrm{mg}$ IV administered every 3 weeks. A control CT scan in February 2017 after 4 doses of pembrolizumab were given showed a good response to therapy with regression of the left previously spiculated left lung mass. PET CT scan later on in April 2017 showed stable nonactive lung nodule at left apex with good response to therapy in superior lobe of left lung, $17 \mathrm{~mm}$ in diameter with SUV of 4.1 against $23 \mathrm{~mm}$ diameter and SUV of 5.1 previously. Injected brain MRI was negative. Immunotherapy was continued and reevaluation was done in august 2017 and disease was stable on CT scan. So decision was also to continue immunotherapy till November 2017 when a PET-CT scan showed a $16 \mathrm{~mm}$ opacity with new nodule in posterior segment of upper lobe suggestive of recurrence with active left hilar adenopathy. An MRI of mediastinum was done and showed a 12-mm mass compatible with the known neoplastic process separated this time from left pulmonary artery and aortic arch, in contrast to initial imaging, with presence of left hilar adenopathy. In front of these new findings and the anatomical separation, decision was to do left lobectomy and patient was operated in January 2018. The final pathology report came positive for a moderately differentiated infiltrating lung adenocarcinoma of acinar and papillary types of $1.7 \mathrm{~cm}$ in diameter with one positive node out of 4 in aorto-pulmonary window and negative other adenopathies in left bronchus and 3 
negative interlobular and hilar nodes. PD-L1 on post-operative tissue was negative using the same Abcam clone. In April 2018, the patient was still off therapy and a total body CT scan done for diffuse whole body pain reported by the patient showed no evidence of recurrent disease. He was diagnosed with a left hip fracture, operated in June 2018, with no evidence of malignancy on bone specimen.

\section{Discussion and Conclusion}

Stage III lung cancer is that marginal state, just at the tip, with still regionally confined disease. An inoperable nonmetastatic lung cancer makes treatment decision challenging to both oncologists and surgeons having to elect between curative and palliative approach.

Overall, 2 different issues are to be considered in both cases: the conversion from an inoperable to operable disease and the negativation of PD-L1 after surgery.

First, the conversion of inoperable to operable disease using immunotherapy is unique.

A recently published article stated that immunotherapy was used in the neoadjuvant setting in operable lung cancer replacing chemotherapy induction [4]. In our cases, the use of immunotherapy was not intended to convert to operable disease but was used as a first-line combination in the first patient and as a second-line therapy in the second one after progression. Both patients had locally advanced stage IIIA disease, deemed to be inoperable due to local extension (enlarged lymph nodes in the barety lodge and the supra-aortic area in the first case, and intimate contact of the primary tumor with left pulmonary artery in the second one). Neoadjuvant chemotherapy is not the standard of care in inoperable stage III lung cancer and to our knowledge, there is no published data on surgical conversion after chemotherapy in inoperable stage III lung cancer, although chemotherapy has been used in this setting for many years. This led us to conclude that the surgical conversion may be due to the use of immunotherapy, a fact that has not been previously described with this novel therapy.

Second, the negativation of PD-L1 status post-systemic therapy is also unique. In fact, previous studies have reported changes in the PD-L1 after anti-cancer treatments $[6,7]$ including concomitant chemo-radiotherapy modality [8,9] and platinum based chemotherapy [10]. However, no studies included any immunotherapy in this setting and no reports were found stating a clear negativation of PD-L1 status (in our cases, from $60 \%$ and $80 \%$ to less than $1 \%$, defined as negative, and more specifically a zero tumor proportion score). We are not sure of the exact time of conversion to negative PD-L1, neither the reason behind it. It might have occurred due to chemotherapy alone or as an immunotherapy related effect, or due to combination/sequencing. If a biopsy was done before immunotherapy in the second case, we could have an idea about the time of negativation. It is also worth to note that the negative results were rechecked by the pathologist in order to avoid a false conclusion.

In front of these multiple observations and especially the conversion to PDL-1 negative status, further studies should be done in stage III inoperable lung cancers to define the exact role of immunotherapy as induction protocol leading to a better outcome in this subcategory of patients.

\section{Acknowledgement}

We would like to thank the patients for giving us their consent to publish the results. 


\section{Statement of Ethics}

Research involving non-routine analyses was approved by the Institutional Review Board at the MOUNT Lebanon hospital and written informed consent obtained from the patient described herein, in accordance with applicable local and international legal and ethical standards, including the Declaration of Helsinki of the World Medical Association.

\section{Disclosure Statement}

The authors have no conflicts of interest to declare.

\section{Funding Sources}

There is no funding to report.

\section{Author Contributions}

AG, RE, LN, SD collected data. AG, RE, SD, SH designed and wrote the manuscript. FN and MR provided relevant scientific advice and revised the manuscript, analyzed and interpreted the patient data regarding clinical presentation, physiopathologic aspects and treatment options. All listed authors contributed to critically revising the article and have approved the final version.

\section{References}

1 What Are the Risk Factors for Lung Cancer? CDC [Internet]. 2019 [cited 2019 Feb 25]. Available from: https://www.cdc.gov/cancer/lung/basic_info/risk_factors.htm.

2 Hellmann MD, Ciuleanu TE, Pluzanski A, Lee JS, Otterson GA, Audigier-Valette C, et al. Nivolumab plus Ipilimumab in Lung Cancer with a High Tumor Mutational Burden. N Engl J Med. 2018 May;378(22):2093104.

3 Reck M, Rodríguez-Abreu D, Robinson AG, Hui R, Csőszi T, Fülöp A, et al. Pembrolizumab versus Chemotherapy for PD-L1-Positive Non-Small-Cell Lung Cancer. N Engl J Med. 2016 Nov;375(19):1823-33.

4 Forde PM, Chaft JE, Smith KN, Anagnostou V, Cottrell TR, Hellmann MD, et al. Neoadjuvant PD-1 Blockade in Resectable Lung Cancer. N Engl J Med. 2018 May;378(21):1976-86.

5 Antonia SJ, Villegas A, Daniel D, Vicente D, Murakami S, Hui R, et al.; PACIFIC Investigators. Durvalumab after Chemoradiotherapy in Stage III Non-Small-Cell Lung Cancer. N Engl J Med. 2017 Nov;377(20):1919-29.

6 Haratake N, Toyokawa G, Tagawa T, Kozuma Y, Matsubara T, Takamori S, et al. Positive Conversion of PD-L1 Expression After Treatments with Chemotherapy and Nivolumab. Anticancer Res. 2017 Oct;37(10):5713-7.

7 Lim SH, Hong M, Ahn S, Choi YL, Kim KM, Oh D, et al. Changes in tumour expression of programmed deathligand 1 after neoadjuvant concurrent chemoradiotherapy in patients with squamous oesophageal cancer. Eur J Cancer. 2016 Jan;52:1-9.

8 Fujimoto D, Uehara K, Sato Y, Sakanoue I, Ito M, Teraoka S, et al. Alteration of PD-L1 expression and its prognostic impact after concurrent chemoradiation therapy in non-small cell lung cancer patients. Sci Rep. 2017 Sep;7(1):11373.

9 Lim YJ, Koh J, Kim S, Jeon S-R, Chie EK, Kim K, et al. Chemoradiation-Induced Alteration of Programmed Death-Ligand 1 and CD8+ Tumor-Infiltrating Lymphocytes Identified Patients With Poor Prognosis in Rectal Cancer: A Matched Comparison Analysis. Int J Radiat Oncol Biol Phys. 2017 Dec;99(5):1216-24.

10 Rojkó L, Reiniger L, Téglási V, Fábián K, Pipek O, Vágvölgyi A, et al. Chemotherapy treatment is associated with altered PD-L1 expression in lung cancer patients. J Cancer Res Clin Oncol. 2018 Jul;144(7):1219-26. 